\title{
High-Resolution Characterization of Liquid-Crystalline [60]Fullerenes Using Solid-State Nuclear Magnetic Resonance Spectroscopy
}

\author{
Sergey V. Dvinskikh, ${ }^{\dagger, »}$ Kazutoshi Yamamoto, ${ }^{\dagger}$ David Scanu, ${ }^{\S}$ Robert Deschenaux,,${ }^{\S}$ and \\ Ayyalusamy Ramamoorthy $*, \dagger$ \\ Biophysics and Department of Chemistry, University of Michigan, Ann Arbor, Michigan 48109-1055, and Institut de \\ Chimie, Université de Neuchâtel, Avenue de Bellevaux 51, Case postale 158, 2009 Neuchâtel, Switzerland
}

Received: April 15, 2008; Revised Manuscript Received: July 5, 2008

Liquid-crystalline materials containing fullerenes are valuable in the development of supramolecular switches and in solar cell technology. In this study, we characterize the liquid-crystalline and dynamic properties of fullerene-containing thermotropic compounds using solid-state natural abundance ${ }^{13} \mathrm{C}$ NMR experiments under stationary and magic angle spinning sample conditions. Chemical shifts spectra were measured in isotropic, liquid-crystalline nematic and smectic $\mathrm{A}$ and crystalline phases using one-dimensional ${ }^{13} \mathrm{C}$ experiments, while two-dimensional separated local-field experiments were used to measure the ${ }^{1} \mathrm{H}-{ }^{13} \mathrm{C}$ dipolar couplings in mesophases. Chemical shift and dipolar coupling parameters were used to characterize the structure and dynamics of the liquid-crystalline dyads. NMR data of fullerene-containing thermotropic liquid crystals are compared to that of basic mesogenic unit and mesomorphic promoter compounds. Our NMR results suggest that the fullerene-ferrocene dyads form highly dynamic liquid-crystalline phases in which molecules rotate fast around the symmetry axis on the characteristic NMR time scale of $\sim 10^{-4} \mathrm{~s}$.

\section{Introduction}

The self-organization in two-dimensional (2D) and threedimensional (3D) space offered by the liquid crystals is an ideal vehicle to explore and control the organization of matter on the nanometer scale. ${ }^{1}$ Design of functional supramolecular mesomorphic materials, which combine the organization behavior of liquid crystals with the properties of the fullerenes, is expected to have a strong impact on the future development of materials science. ${ }^{1}$ Because of the unique photophysical and electrochemical properties of $\mathrm{C}_{60},{ }^{2}$ the latter was combined with ferrocene, ${ }^{3}$ tetrathiafulvalene (TTF) ${ }^{4}$ or oligophenylenevinylene $(\mathrm{OPV})^{5}$ and a mesomorphic promoter to develop liquidcrystalline donor-acceptor dyads for which photoinduced electron and/or energy transfer could be achieved. Such dyads are appealing for the design of supramolecular switches ${ }^{2}$ and in solar cell technology. ${ }^{6}$ The above liquid crystals gave rise to smectic B and/or smectic A phases in agreement with the nature and structure of the liquid-crystalline addends grafted onto $\mathrm{C}_{60}$. On the other hand, no studies on the high-resolution structural and dynamical characterization of these molecules have been reported so far. Such investigations would better our understanding of the general properties of these materials. In this study, we use high-resolution solid-state NMR (nuclear magnetic resonance) techniques to characterize the liquid-crystalline and dynamic properties of fullerene-containing thermotropic liquid crystals (compounds $\mathbf{3}$ and $\mathbf{4}$ ), liquid-crystalline promoter (compound 2), and mesogenic unit (compound 1) (Figure 1). The peralkylated ferrocene unit was oxidized to see a possible influence of the charge on the NMR properties.

* To whom correspondence should be addressed. Tel: 734-647-6572. E-mail: ramamoor@umich.edu.

${ }^{\dagger}$ University of Michigan.

¥ Present address: Division of Physical Chemistry and Industrial NMR Centre, Department of Chemistry, Royal Institute of Technology, SE-100 44 Stockholm, Sweden.

§ Université de Neuchâtel.
NMR spectroscopy is a powerful experimental tool to investigate the structure and dynamics of crystalline, noncrystalline, and nonsoluble materials at atomistic-level resolution. ${ }^{7,8}$ Particularly, it is impressive that the applications of solid-state NMR spectroscopy are not limited by the molecular size, solubility, or phase of system under study. Therefore, highresolution information on molecular structure and mobility can be obtained from isotropic, solid, and liquid-crystalline phases of a variety of materials. ${ }^{7,9,10}$ Carbon-13 NMR spectroscopy has several advantages for studying mesophases. Isotropic "solutionlike" carbon-13 chemical shift spectra with excellent signal-tonoise ratios can be obtained even from nonisotropic samples and without the need for an isotopic enrichment in the sample. Because ${ }^{13} \mathrm{C}$ chemical shift resonances from chemically nonequivalent sites are typically well-resolved, it is easy to identify the chemical groups of the molecule under study. In addition, simple one-dimensional (1D) ${ }^{13} \mathrm{C}$ isotropic chemical shift measurements can reveal the crystalline and amorphous properties of the sample. Anisotropic spin interactions, such as chemical shifts or dipolar couplings, observed in 1D or 2D spectra provide information on molecular ordering, structure, and phase transitions. ${ }^{7,9-15}$ The experiments were carried out on both stationary and magic angle spinning (MAS) experimental conditions.

\section{Experimental Section}

2.1. Materials. The compounds under investigation were prepared by adapting previously described procedures; ${ }^{3}$ the syntheses will be reported in a subsequent paper. The phase transition temperatures are reported in Figure 2.

2.2. NMR Experiments. NMR experiments were performed at a magnetic field of $9.4 \mathrm{~T}$ on a Chemagnetics/Varian Infinity $400 \mathrm{MHz}$ spectrometer at the resonance frequencies of 400 and $100 \mathrm{MHz}$ for protons $\left({ }^{1} \mathrm{H}\right)$ and carbons $\left({ }^{13} \mathrm{C}\right)$, respectively. A 5 $\mathrm{mm}$ double-resonance MAS probe was used. The measurements were performed on samples either at stationary or MAS 


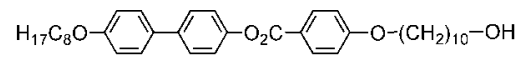

\section{Compound-1}

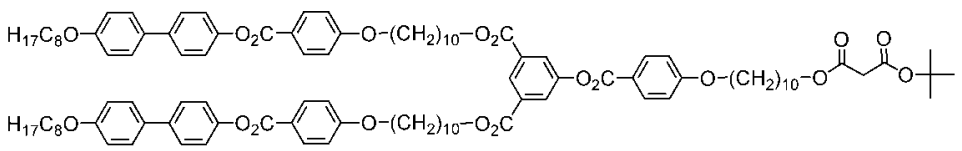

Compound-2
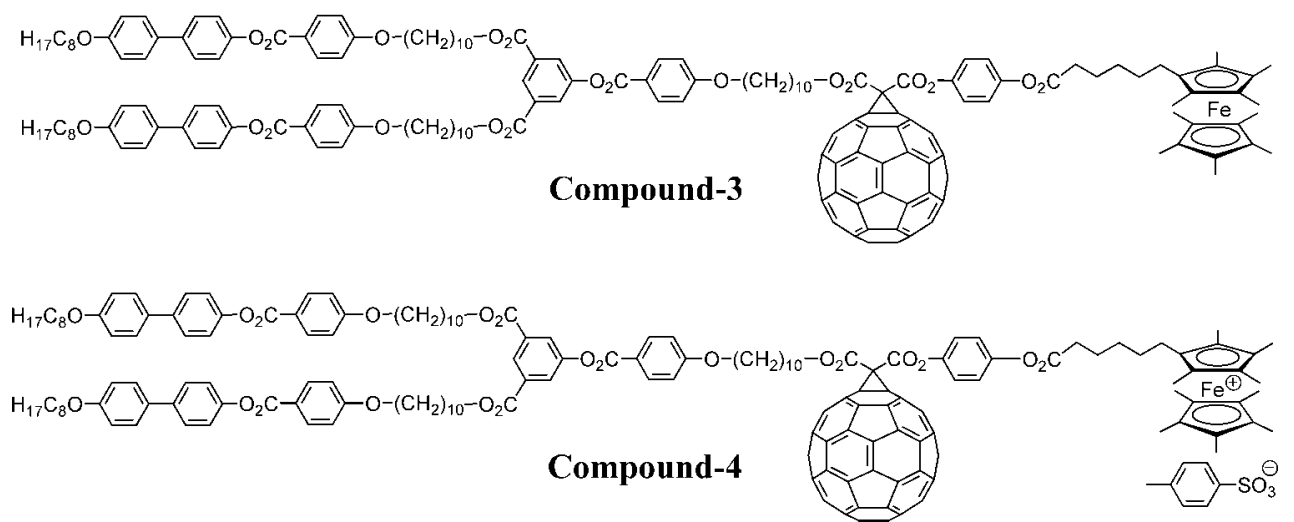

Figure 1. Molecular structure of thermotropic liquid-crystalline samples investigated in this study.

Compound 1: Solid $-128^{\circ} \mathrm{C}-$ smectic $\mathrm{C}-142^{\circ} \mathrm{C}-$ nematic $-176^{\circ} \mathrm{C}-$ isotropic

Compound 2: Solid $-119^{\circ} \mathrm{C}-$ smectic $\Lambda-141^{\circ} \mathrm{C}-$ nematic $-143^{\circ} \mathrm{C}$ - isotropic

Compound 3: Solid $-80-90^{\circ} \mathrm{C}-$ smectic B $-128^{\circ} \mathrm{C}-$ smectic $\mathrm{A}-138^{\circ} \mathrm{C}-$ isotropic

Compound 4: Solid $-80-90^{\circ} \mathrm{C}-$ smectic B $-124^{\circ} \mathrm{C}-$ smectic $\mathrm{A}-140^{\circ} \mathrm{C}$ - isotropic

Figure 2. Phase transition temperatures of the liquid-crystalline compounds.

experimental conditions with a spinning frequency up to $10 \mathrm{kHz}$. For the signal enhancement by Hartmann-Hahn cross-polarization $(\mathrm{CP}),{ }^{16-18}$ the radio frequency (rf) fields with nutation frequencies of $40-60 \mathrm{kHz}$ and contact times of $1-3 \mathrm{~ms}$ were applied. Heteronuclear dipolar decoupling during the detection period was achieved by using a $70 \mathrm{kHz}$ TPPM (two pulse phase modulation) irradiation. ${ }^{19}$ Carbon-13 chemical shifts were referenced to the MAS spectra obtained for solid adamantane sample. ${ }^{20}$ Macroscopically aligned samples in mesophase were prepared by slowly cooling at a rate less than $0.1 \mathrm{~K} / \mathrm{min}$ from the isotropic phase, while the sample was in the $9.4 \mathrm{~T}$ magnetic field of the $400 \mathrm{MHz}$ NMR spectrometer. Unoriented powderlike samples were used for MAS experiments in solid or smectic phases. Random powder distribution of crystallites or liquidcrystalline domains was ensured by using a sample freshly packed in the MAS rotor or by cooling a sample from its isotropic phase to smectic phase outside the magnetic field. Numerical simulations of NMR spectra were carried out using the SIMPSON simulation package. ${ }^{21}$

2.3. Liquid-Crystalline Properties. Transition temperatures were determined with a differential scanning Mettler DSC 822 calorimeter, under $\mathrm{N}_{2} / \mathrm{He}$, at a rate of $10{ }^{\circ} \mathrm{C} \min ^{-1}$; the instrument was calibrated with In (onset temperature, $156.6 \pm$ $0.3{ }^{\circ} \mathrm{C}$; heat flow, $28.45 \pm 0.6 \mathrm{~J} \mathrm{~g}^{-1}$ ). The textures of the liquidcrystalline phases were observed using a Zeiss-Axioskop polarizing microscope equipped with a Linkam-THMS-600 variable-temperature stage, under $\mathrm{N}_{2}$.

\section{Results and Discussion}

3.1. ${ }^{13}$ C Isotropic Chemical Shift Spectra. Carbon-13 chemical shift spectra of compounds $\mathbf{1}$ and $\mathbf{2}$ obtained in
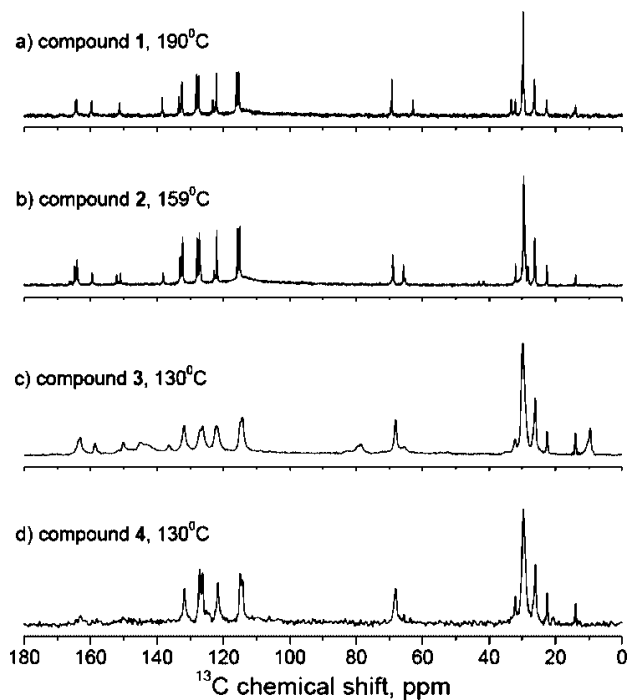

Figure 3. Carbon-13 isotropic chemical shift spectra of liquidcrystalline samples. Spectra of compounds 1 (a) and $\mathbf{2}$ (b) were measured in the isotropic fluid phase under stationary sample conditions, while the spectra of compounds 3 (c) and 4 (d) were obtained in the smectic phase under an $8 \mathrm{kHz}$ MAS.

isotropic liquid state of static samples are shown in Figure 3a,b, respectively. Because the corresponding isotropic phase spectra of compounds $\mathbf{3}$ and $\mathbf{4}$ exhibited broad peaks (see, for example, the spectrum of compound $\mathbf{3}$ in Figure 5), we have included MAS spectra of these two compounds recorded in their mesophases where much narrower resonances were observed (Figure 3c,d). There was no significant difference in the isotropic chemical shift values measured from isotropic state and mesophase as demonstrated for compound 2 in Figure 4. While there was no attempt made to unambiguously assign all carbon13 isotropic signals, it is obvious from the chemical shift values that the peaks in the 150-165 and 115-140 ppm ranges belong to the carbonyl and aromatic carbons, respectively. ${ }^{22}$ Peaks in the $64-70$ and $20-35 \mathrm{ppm}$ ranges result from the $\alpha$-methylene 
a) $190^{\circ} \mathrm{C}$

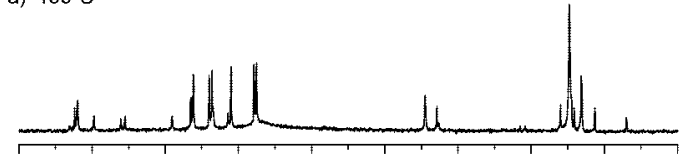

b) $140^{\circ} \mathrm{C}$
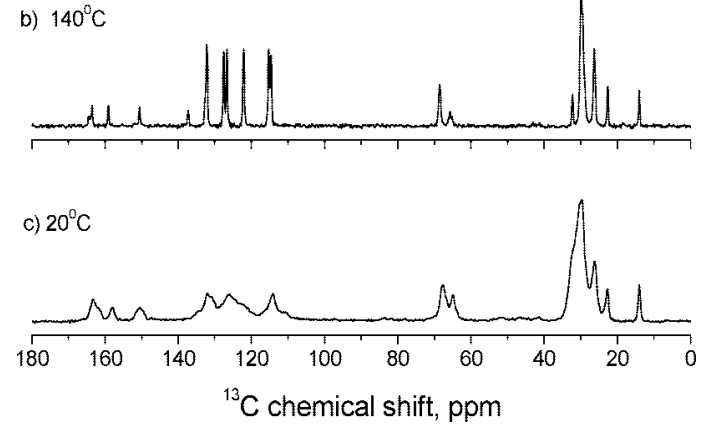

Figure 4. Carbon-13 chemical shift spectra of compound $\mathbf{2}$ in isotropic phase at $190{ }^{\circ} \mathrm{C}(\mathrm{a})$, mesophase at $140{ }^{\circ} \mathrm{C}(\mathrm{b})$, and solid phase at $20^{\circ} \mathrm{C}$ (c). Spectra $\mathrm{b}$ and $\mathrm{c}$ were obtained under an $8 \mathrm{kHz}$ MAS condition. There is no significant difference in the chemical shift values measured from isotropic phase (spectrum a) and mesophase (spectrum b) of the compound.

a) $165^{\circ} \mathrm{C}$
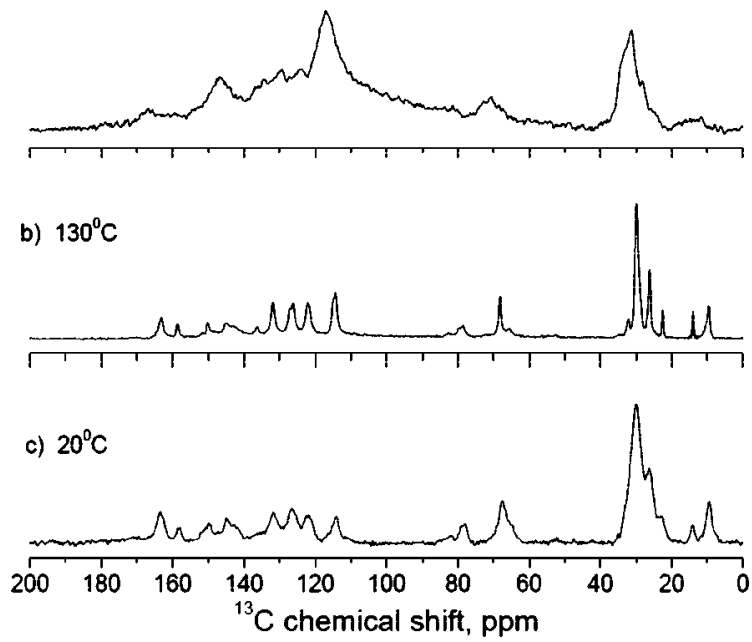

Figure 5. Carbon- 13 chemical shift spectra of compound $\mathbf{3}$ in isotropic phase at $165^{\circ} \mathrm{C}(\mathrm{a})$, smectic phase at $130{ }^{\circ} \mathrm{C}(\mathrm{b})$, and solid phase at 20 ${ }^{\circ} \mathrm{C}$ (c). Spectra b and c were obtained under an $8 \mathrm{kHz}$ MAS condition.

carbons and the rest of the methylene groups in the side chains, respectively.

Compound 3 exhibited ${ }^{13} \mathrm{C}$ isotropic chemical shift peaks around 144, 80, and $10 \mathrm{ppm}$ (Figure 3c), which were not observed in the spectra of other liquid-crystalline compounds. The broad resonances $\sim 80$ and $\sim 144$ ppm correspond to signals from sp2 and sp3 carbons of $\mathrm{C} 60$, respectively, ${ }^{4,23}$ while the origin of the peak at $10 \mathrm{ppm}$ is not known. (C60 signals were not observed in a sample of compound $\mathbf{4}$ due to a low signalto-noise ratio.)

${ }^{13} \mathrm{C}$ MAS spectra recorded in the solid phase exhibited broad spectral lines, characteristic of the amorphous state (ca. Figures $4 c, 5 c$, and $6 b$ ). Narrow ${ }^{13} \mathrm{C}$ chemical shift spectral lines in the mesophases are characteristic of liquid-crystalline nature of the sample with fast anisotropic molecular rotations on the typical time scale of spin interactions $\left(10^{-4} \mathrm{~s}\right)$. Somewhat broad peaks were observed from the mesophases of compounds $\mathbf{3}$ and $\mathbf{4}$ as compared to compound $\mathbf{2}$, which could be attributed to slow a) $130^{\circ} \mathrm{C}$
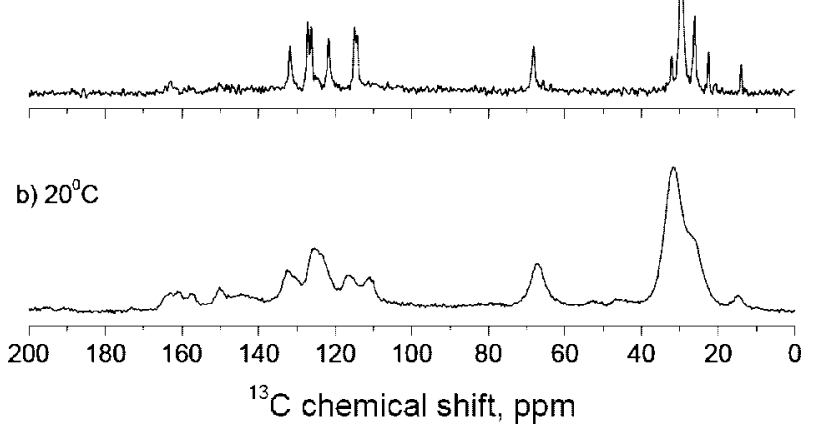

Figure 6. Carbon-13 isotropic chemical shift spectra of compound 4 obtained under an $8 \mathrm{kHz}$ MAS: (a) smectic phase at $130{ }^{\circ} \mathrm{C}$ and (b) solid phase at $20^{\circ} \mathrm{C}$.
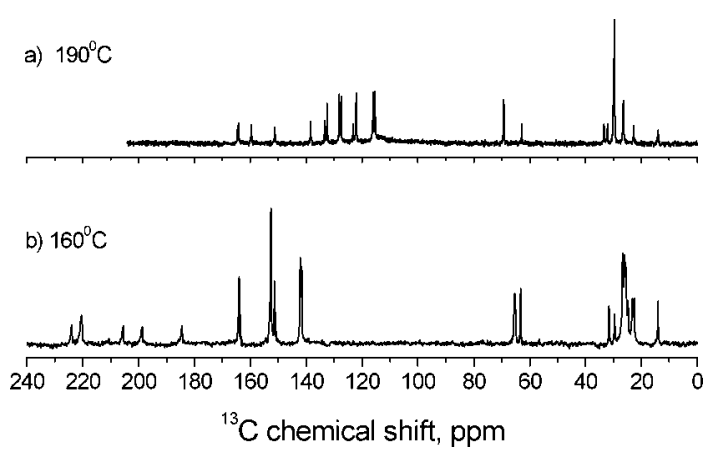

Figure 7. Comparison of carbon-13 chemical shift spectra of compound $\mathbf{1}$ in isotropic phase at $190{ }^{\circ} \mathrm{C}$ (a) and in macroscopically oriented nematic phase at $160{ }^{\circ} \mathrm{C}(\mathrm{b})$. A downfield shift of the aromatic and a small upfield shift of aliphatic resonances are observed in oriented mesophase as compared to the spectrum in isotropic phase.

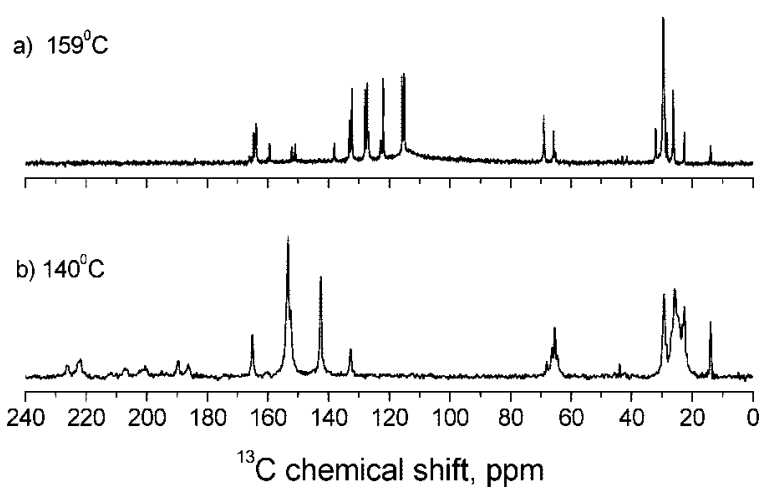

Figure 8. Carbon-13 chemical shift spectra of compound $\mathbf{2}$ in isotropic phase at $159^{\circ} \mathrm{C}$ (a) and in macroscopically oriented mesophase at 140 ${ }^{\circ} \mathrm{C}$ (b). A downfield shift of the aromatic and a small upfield shift of aliphatic resonances are observed in oriented mesophase as compared to the spectrum in isotropic phase.

and/or restricted motional effects. These observations are likely due to the steric restrictions for the molecular rotations imposed by the presence of the attached fullerene in these compounds.

3.2. ${ }^{13} \mathrm{C}$ Chemical Shift Spectra of Static Macroscopically Oriented Samples. 3.2.1. One-Dimensional ${ }^{13} \mathrm{C}$ Chemical Shift Spectra. The samples of compounds $\mathbf{1}$ and $\mathbf{2}$ were macroscopically aligned, with the director uniformly oriented with the respect to the magnetic field, by slowly cooling the samples from their isotropic phases in the presence of the static magnetic field of the NMR spectrometer. Carbon-13 chemical shift spectra of aligned samples along with their isotropic phase spectra are displayed in Figures 7 and 8 for compounds $\mathbf{1}$ and $\mathbf{2}$, respectively. While no unambiguous correspondence between 


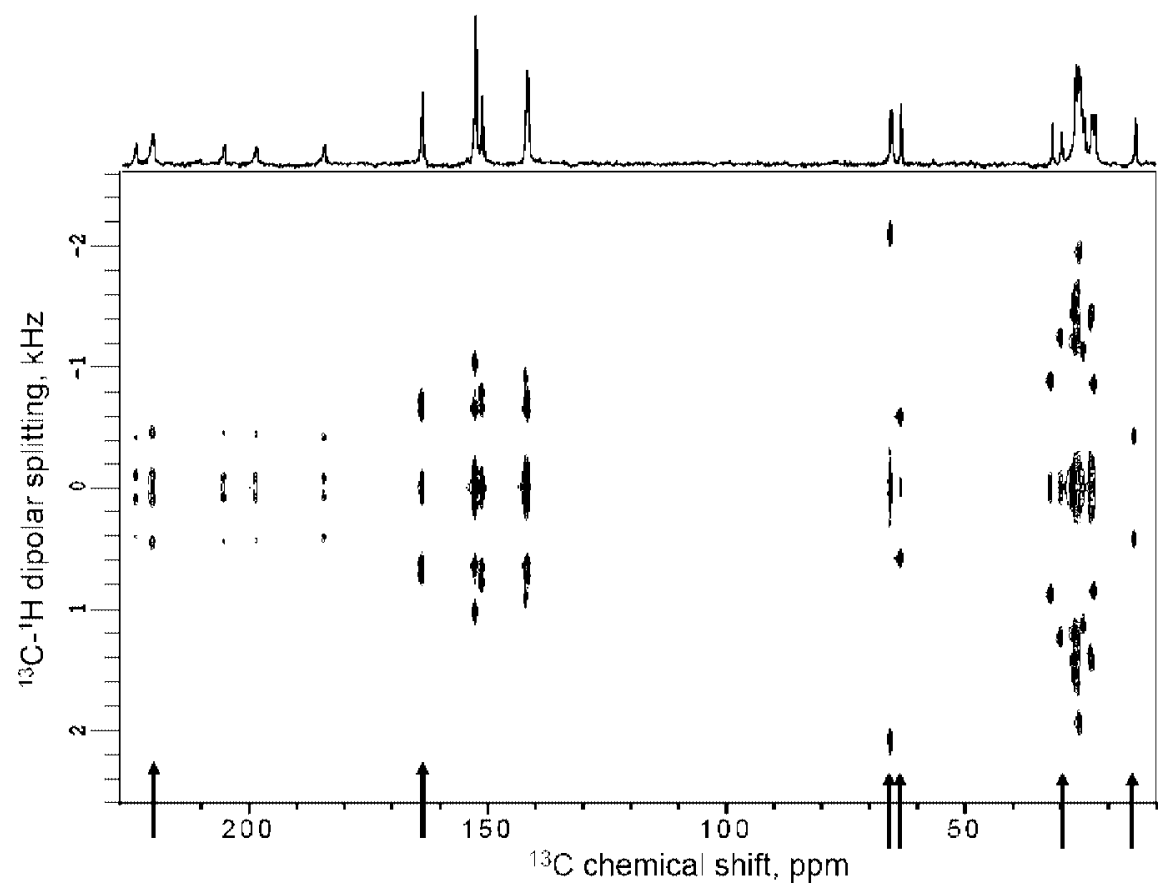

Figure 9. Two-dimensional PDLF spectrum of compound $\mathbf{1}$ in macroscopically oriented nematic phase at $160{ }^{\circ} \mathrm{C}$. A $1 \mathrm{D}{ }^{13} \mathrm{C}$ chemical shift spectrum is displayed on top of the 2D spectrum. Arrows indicate frequency positions of the cross-sections displayed in Figure 10.

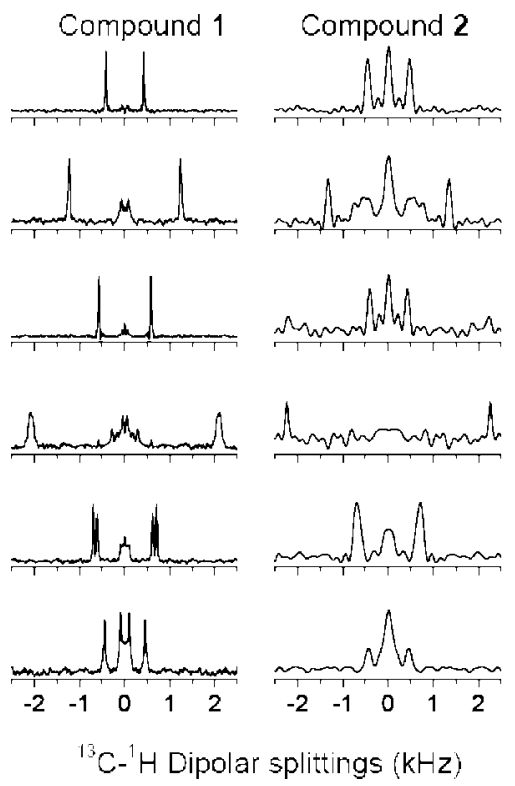

Figure 10. Selective ${ }^{1} \mathrm{H}-{ }^{13} \mathrm{C}$ dipolar coupling spectral slices extracted from 2D PDLF spectra of compounds $\mathbf{1}$ (left column) and $\mathbf{2}$ (right column) in oriented mesophase at 160 and $140{ }^{\circ} \mathrm{C}$, respectively. Slices were taken from the corresponding $2 \mathrm{D}$ spectra at the chemical shift positions (from top to bottom) 14, 30, 63, 65, 164, and $221 \mathrm{ppm}$ as indicated in Figure 9 for compound 1.

resonances in isotropic and mesophases was accomplished in this study, it is obvious that the observed chemical shift values for the aromatic resonances in the mesophases $\left(\delta_{\|}\right)$are shifted downfield with respect to the isotropic peaks (for different peaks the shifts are in the range of $25-30 \mathrm{ppm}$ ). Significant downfield shift of the aromatic and small upfield of aliphatic resonances is typical of thermotropic mesophases of calamitic molecules. ${ }^{24}$ Such an observation confirms that molecules align with their long axis parallel to the magnetic field direction due to the positive bulk magnetic susceptibility. With the assumption that the molecular rotation axis is approximately parallel to the plane of the aromatic ring, the least shielded principal chemical shift anisotropy component, $\left(\delta_{33}\right)$, parallel to the normal of the aromatic ring plane, ${ }^{22}$ is averaged to zero. The frequency value of the component $\left(\delta_{\|}\right)$is determined by the two in-plane components $\delta_{11}$ and $\delta_{22}$ (with typical values in the ranges of $220-230$ and $150-160 \mathrm{ppm}$, respectively ${ }^{25}$ ) that are partially averaged by the molecular rotations. The anisotropic chemical shift values are very similar in these two samples, suggesting the similarity of the molecular orientational order. This is further confirmed by the measurement of the dipolar coupling values as described below in section 3.3.

Two fullerene-containing samples, compounds $\mathbf{3}$ and $\mathbf{4}$, do not exhibit nematic phase and do not orient macroscopically in the magnetic field of the spectrometer. This property of these compounds can be attributed to their high viscosity of the smectic phase. It should be noted that these samples preserve high viscosity even in the isotropic phase, where broad ${ }^{13} \mathrm{C}$ spectral lines were observed.

3.3. Two-Dimensional ${ }^{1} \mathbf{H}-{ }^{13} \mathbf{C}$ Separated Local-Field (SLF) Spectra. 3.3.1. SLF Spectra of Oriented Samples. Twodimensional SLF experiments correlate the chemical shift interaction with the heteronuclear dipolar couplings. ${ }^{7,26}$ A variety of laboratory frame and rotating frame techniques ${ }^{27,28}$ have been developed and utilized to determine high-resolution structures of chemical and biological molecules on aligned samples ${ }^{26,29}$ as well as on samples under MAS. ${ }^{26,30}$ Our recent studies reported that some of the rotating frame SLF pulse sequences like PISEMA (polarization inversion spin exchange at the magic angle), ${ }^{26}$ BB-PISEMA (broadband-PISEMA), and HIMSELF (heteronuclear isotropic mixing spin exchange via local field) provide very high-resolution heteronuclear dipolar coupling spectral lines, whereas laboratory frame SLF pulse sequences like PDLF 27,31 in general are not efficient in suppressing homonuclear ${ }^{1} \mathrm{H}-{ }^{1} \mathrm{H}$ dipolar couplings; therefore, the resultant SLF spectra are not that high in spectral resolution for rigid solids. ${ }^{32}$ In addition, our studies showed that the laboratory frame SLF sequence, PDLF (proton-encoded SLF), is useful for the accurate measurement of heteronuclear dipolar couplings from dynamical semisolids like liquid crystals. ${ }^{32}$ Therefore, in this 


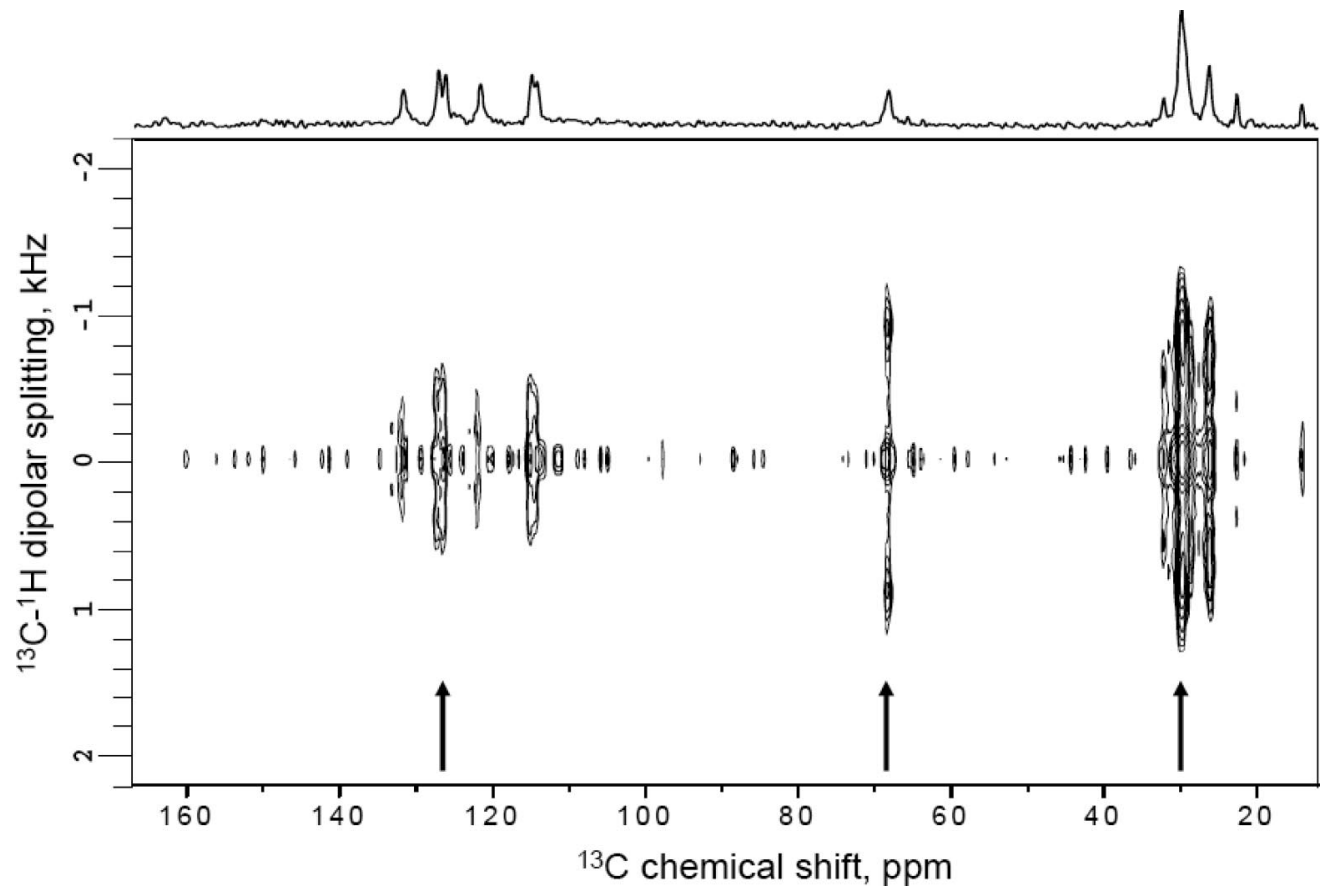

Figure 11. Two-dimensional R-PDLF MAS spectrum of compound 4 in smectic A phase at $130{ }^{\circ} \mathrm{C}$. A $1 \mathrm{D}{ }^{13} \mathrm{C}$ MAS spectrum is displayed on the top. Arrows indicate positions of the cross-sections displayed in Figure 12.

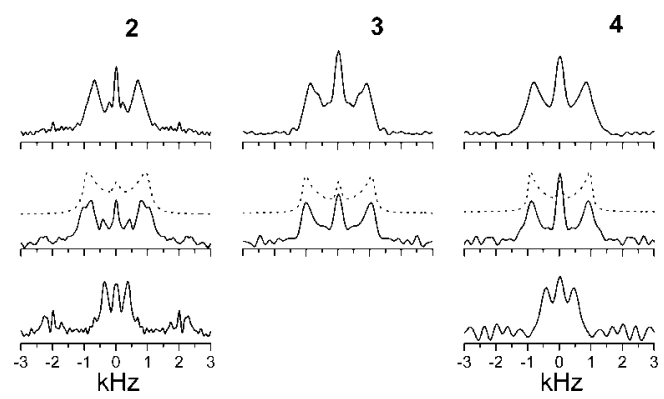

Figure 12. Selected dipolar coupling slices from the 2D R-PDLF spectra of compounds $\mathbf{2}$ (left column), $\mathbf{3}$ (middle), and $\mathbf{4}$ (right) samples in the mesophase at 140,130 , and $130{ }^{\circ} \mathrm{C}$, respectively. Spectral slices were taken from the corresponding $2 \mathrm{D}$ spectra at the chemical shift positions 28 (top row), 68 (middle), and $128 \mathrm{ppm}$ (bottom) as also indicated in Figure 11 for compound 4. Calculated ${ }^{1} \mathrm{H}-{ }^{13} \mathrm{C}$ dipolar coupling spectra for $\alpha$-carbon groups are shown in dashed lines.

study, we apply the PDLF pulse sequence to characterize the liquid-crystalline compounds at a higher resolution using the experimentally measured $\mathrm{C}-\mathrm{H}$ dipolar couplings. In a 2D PDLF spectrum, the ${ }^{1} \mathrm{H}-{ }^{13} \mathrm{C}$ dipolar coupling split doublets in the first (indirectly detected) frequency dimension are correlated with the ${ }^{13} \mathrm{C}$ chemical shift interaction in the second (direct) frequency dimension. A contour plot 2D PDLF spectrum of compound 1 is shown in Figure 9.

For most of the carbon sites, well-resolved doublets resulted from the ${ }^{1} \mathrm{H}-{ }^{13} \mathrm{C}$ dipolar couplings are observed. As typical of thermotropic mesophases of calamitic molecules, ${ }^{11}$ larger splittings are observed for aliphatic carbons than for the aromatic core sites. The $\alpha$-methylene carbons exhibit the largest ${ }^{1} \mathrm{H}-{ }^{13} \mathrm{C}$ dipolar splittings. ${ }^{1} \mathrm{H}-{ }^{13} \mathrm{C}$ dipolar splittings observed for the aromatic carbons are small due to specific orientation of the $\mathrm{C}-\mathrm{H}$ bond with respect to molecular axis (see below). The dipolar splittings observed for the carbonyl carbons are due to the weak coupling with the remote protons in the adjacent aromatic rings.
The ${ }^{1} \mathrm{H}-{ }^{13} \mathrm{C}$ dipolar splitting is given by

$$
\Delta v=\left|2 S_{\mathrm{CH}} k d_{\mathrm{CH}}\right|
$$

where $S_{\mathrm{CH}}$ is the local order parameter of the $\mathrm{C}-\mathrm{H}$ bond vector, $d_{\mathrm{CH}}$ is the dipolar coupling constant for a rigid molecule, and $k$ $\approx 0.42$ is the scaling factor due to the homonuclear decoupling multiple pulse sequence BLEW48 ${ }^{33}$ used in the 2D PDLF experiment. The experimental scaling factor $k \approx 0.40$ estimated from the scaling of the proton resonance offset frequency is close to the theoretical value (0.42). Any contributions from carbon-proton $J$ couplings are neglected. The local order parameter describes the averaged orientation of the $\mathrm{C}-\mathrm{H}$ vector in the laboratory frame, which is defined by the direction of the external magnetic field. With the director parallel to the external magnetic field, the order parameter is given by

$$
S_{\mathrm{CH}}=\left\langle d_{00}^{2}\left(\cos \vartheta_{\mathrm{PN}}\right)\right\rangle
$$

where $\vartheta_{\text {PN }}$ is the angle between the internuclear vector $\mathbf{P}$ and the liquid crystalline director $\mathbf{N}$, and $d_{00}^{2}\left(\cos \vartheta_{\mathrm{PN}}\right)=\left(3 \cos ^{2}\right.$ $\left.\vartheta_{\mathrm{PN}}-1\right) / 2$ is the reduced Wigner matrix element. For the directly bonded $\mathrm{C}$ and $\mathrm{H}$ atoms, $d_{\mathrm{CH}} \approx 21.5 \mathrm{kHz}$ and $S_{\mathrm{CH}}$ corresponds to the $\mathrm{C}-\mathrm{H}$ bond order parameter. The local order parameter in the rigid molecular fragments can be connected to the conventional molecular order parameter $S$ of the mesophase via

$$
S_{\mathrm{CH}}=S \times d_{00}^{2}\left(\cos \vartheta_{\mathrm{PN}}\right)
$$

where $\vartheta_{\mathrm{PN}}$ is the angle between the bond vector and the molecular symmetry axis. The bond order parameter $S_{\mathrm{CH}}$ for the aromatic sites is typically small in the calamitic liquid crystals with the rigid aromatic core. This is because the angle $\vartheta_{\mathrm{PN}}$ is in vicinity of the magic angle value of $54.7^{\circ}$, where the value of the Wigner matrix element is close to zero. The exception is however expected for the single $\mathrm{C}-\mathrm{H}$ pair in junction aromatic ring (for compounds $\mathbf{2}, \mathbf{3}$, and 4 ) where the $\mathrm{CH}$ vector is virtually parallel to the molecular symmetry axis. Unfortunately, the corresponding ${ }^{13} \mathrm{C}$ signal from this single site 
is weak and overlaps with the signals from other multiple aromatic sites. Therefore, it was not resolved in the spectra reported in this study.

In Figure 9, selected ${ }^{1} \mathrm{H}-{ }^{13} \mathrm{C}$ dipolar coupling spectral slices extracted from the 2D PDLF spectrum of compound $\mathbf{1}$ are shown and compared to corresponding spectral slices of compound 2 . It is obvious that the dipolar splitting frequencies and, hence, the order parameters, are comparable in two samples. The bond order parameters for $\alpha$-methylene carbon, exhibiting largest dipolar splitting frequency, and for the terminal methyl group of compound 1 are estimated to be $S_{\mathrm{CH}} \approx 0.24$ and 0.050 , respectively. Corresponding values for compound $\mathbf{2}$ are 0.26 and 0.056. Thus, the molecular ordering is similar (as concerning the time scale and motional amplitudes) for these two compounds in spite of the difference in the molecular weight and structure. Somewhat larger line widths in ${ }^{1} \mathrm{H}-{ }^{13} \mathrm{C}$ dipolar coupling spectra for compound $\mathbf{2}$ are due presumably to less uniform director orientation; it should be noted that large line widths in the $1 \mathrm{D}{ }^{13} \mathrm{C}$ chemical shift spectra were also observed. It could also be possible that the compound $\mathbf{2}$ may exist in the smectic A phase at this temperature that could result in some line broadening.

3.3.2. MAS SLF Spectra of Powder Samples. Magic angle sample spinning SLF experiments were performed in powderlike samples with random director orientation. To ensure powder distribution, samples were prepared by cooling from isotropic phase outside the magnet of the NMR spectrometer. The heteronuclear ${ }^{1} \mathrm{H}-{ }^{13} \mathrm{C}$ dipolar couplings in the powder samples of the liquid crystalline compounds under study were measured using the 2D R-PDLF type SLF MAS experiments. ${ }^{34}$ A contour plot 2D R-PDLF spectrum of compound $\mathbf{4}$ is shown in Figure 11. A few selected ${ }^{1} \mathrm{H}-{ }^{13} \mathrm{C}$ dipolar coupling spectral slices are shown in Figure 12. In agreement with the results from static 2D PDLF spectra of compounds $\mathbf{1}$ and 2 , larger ${ }^{1} \mathrm{H}-{ }^{13} \mathrm{C}$ splittings are seen for the methylene carbons than for the aromatic carbons. The widths of the ${ }^{1} \mathrm{H}-{ }^{13} \mathrm{C}$ dipolar coupling spectral pattern are also of similar magnitude for all of these three samples. In the limit of the negligible line broadening relaxation effects, the frequency separation between the maxima in the dipolar coupling powder pattern is given by the scaled motionally averaged dipolar coupling

$$
\Delta v=\left|k_{\mathrm{R}}<d_{\mathrm{CH}}>\right|=\left|k_{\mathrm{R}} S_{\mathrm{CH}} d_{\mathrm{CH}}\right|
$$

where $k_{\mathrm{R}} \approx 0.32$ is the theoretical scaling factor of the recoupling sequence $\mathrm{R} 18^{7}{ }_{1}$ applied in this experiment. ${ }^{35}$ In practice, finite line broadening may influence the dipolar splitting frequency. Numerical calculations of selected dipolar coupling spectra were performed to estimate the dipolar couplings $\left\langle d_{\mathrm{CH}}\right\rangle$ values by fitting the calculated spectra to experimental line shapes. Calculated ${ }^{1} \mathrm{H}-{ }^{13} \mathrm{C}$ dipolar coupling spectra for $\alpha$-carbon group are included in Figure 12. Corresponding $\mathrm{C}-\mathrm{H}$ bond order parameters $S_{\mathrm{CH}}$ are estimated to be $0.28,0.31$, and 0.29 for compounds $\mathbf{2}, \mathbf{3}$, and $\mathbf{4}$, respectively, practically similar within experimental errors. The order parameter for compound $\mathbf{2}$ is also comparable with that obtained from the dipolar coupling spectra of static samples. Slight deviations in the order parameters for compound $\mathbf{2}$ obtained by two SLF methods can be attributed to the effects of $J$ coupling $\left(J_{\mathrm{CH}} \approx 140 \mathrm{~Hz}\right)$ on the observed spectral splittings in static samples ( $J$ couplings are not recoupled by $\mathrm{R}$ sequence in the R-PDLF experiment) and to the uncertainty in the dipolar coupling scaling factors.

\section{Conclusions}

NMR data confirmed the results from other techniques that highly dynamic liquid-crystalline phases are formed by fullerene-ferrocene dyads. The degree of the molecular ordering is similar to that of the low molecular weight analogues. The high viscosity of the fullerene-ferrocene dyads in liquidcrystalline state prevents their macroscopic orientation in the magnetic field. It is concluded from the ${ }^{13} \mathrm{C}$ NMR spectral line shapes that the molecules in the mesophase undergo rotation around the symmetry axis fast on the characteristic NMR time scale of $\sim 10^{-4} \mathrm{~s}$ as defined by the magnitude of the anisotropic spin interactions. In the case of compounds $\mathbf{3}$ and $\mathbf{4}$, the restricted rotation, due to the steric constraints, is also not excluded. More detailed information on the geometry and the time scale can be obtained by the measurement of the shapes of the dipolar ${ }^{13} \mathrm{C}-{ }^{1} \mathrm{H}$ and ${ }^{13} \mathrm{C}$ chemical shift anisotropy spectra.

Acknowledgment. This work was supported by research funds to A.R.'s laboratory. R.D. thanks the Swiss National Science Foundation (Grant 200020-119648) for financial support.

\section{References and Notes}

(1) Goodby, J. W.; Saez, I. M.; Cowling, S. J.; Görtz, V.; Draper, M.; Hall, A. W.; Sia, S.; Cosquer, G.; Lee, S.-E.; Raynes, E. P. Angew. Chem., Int. Ed. 2008, 47, 2754-2787.

(2) (a) Martín, N. Chem. Commun. 2006, 2093-2104. (b) Martín, N.; Sánchez, L.; Herranz, M. A.; Illescas, B.; Guldi, D. M. Acc. Chem. Res. 2007, 40, 1015-1024.

(3) (a) Even, M.; Heinrich, B.; Guillon, D.; Guldi, D. M.; Prato, M.; Deschenaux, R. Chem. Eur. J. 2001, 7, 2595-2604. (b) Campidelli, S.; Vázquez, E.; Milic, D.; Prato, M.; Barberá, J.; Guldi, D. M.; Marcaccio, M.; Paolucci, D.; Paolucci, F.; Deschenaux, R. J. Mater. Chem. 2004, 14, 1266-1272. (c) Campidelli, S.; Séverac, M.; Scanu, D.; Deschenaux, R.; Vázquez, E.; Milic, D.; Prato, M.; Carano, M.; Marcaccio, M.; Paolucci, F.; Aminur Rahman, G. M.; Guldi, D. M. J. Mater. Chem. 2008, 18, 15041509.

(4) Allard, E.; Oswald, F.; Donnio, B.; Guillon, D.; Delgado, J. L.; Langa, F.; Deschenaux, R. Org. Lett. 2005, 7, 383-386.

(5) Campidelli, S.; Deschenaux, R.; Eckert, J.-F.; Guillon, D.; Nierengarten, J.-F. Chem. Commun. 2002, 656-657.

(6) (a) Nierengarten, J.-F.; Eckert, J.-F.; Nicoud, J.-F.; Ouali, L.; Krasnikov, V.; Hadziioannou, G. Chem. Commun. 1999, 617-618. (b) Eckert, J.-F.; Nicoud, J.-F.; Nierengarten, J.-F.; Liu, S.-G.; Echegoyen, L.; Barigelletti, F.; Armaroli, N.; Ouali, L.; Krasnikov, V.; Hadziioannou, G. J. Am. Chem. Soc. 2000, 122, 7467-7479.

(7) Schmidht-Rohr, K.; Spiess, H. W. Multidimensional Solid-State NMR and Polymers; Academic Press: San Diego, 1994.

(8) Ramamoorthy, A., Ed. NMR Spectroscopy of Biological Solids; Taylor \& Francis: New York, 2006.

(9) Dong, R. Nuclear Magnetic Resonance of Liquid Crystals: Partially Ordered Systems, 2nd ed.; Springer-Verlag: New York, 1997.

(10) Ramamoorthy, A., Ed. Thermotropic Liquid Crystals: Recent Advances; Springer: Dordrecht, The Netherlands, 2007.

(11) Fung, B. M. Prog. Nucl. Magn. Reson. Spectrosc. 2002, 41, 171186.

(12) Dvinskikh, S. V.; Sandström, D.; Zimmermann, H.; Maliniak, A. Prog. Nucl. Magn. Reson. Spectrosc. 2006, 48, 85-107.

(13) (a) Havlin, R. H.; Park, G. H. J.; Mazur, T.; Pines, A. J. Am. Chem. Soc. 2003, 26, 7998-8006. (b) Havlin, R. H.; Park, G. H. J.; Pines, A. J. Magn. Reson. 2002, 157, 163-169. (c) Long, H. W.; Luzar, M.; Gaede, H. C.; Larsen, R. G.; Pines, A.; Crawford, G. P. J. Phys. Chem. 1995, 99, 11989-11993.

(14) (a) Narasimhaswamy, T.; Monette, M.; Lee, D. K.; Ramamoorthy, A. J. Phys. Chem. A 2005, 109, 19696-19703. (b) Narasimhaswamy, T.; Lee, D. K.; Somanathan, N.; Ramamoorthy, A. Chem. Mater. 2005, 17, 4567-4569. (c) Narasimhaswamy, T.; Lee, D. K.; Yamamoto, K.; Somanathan, N.; Ramamoorthy, A. J. Am. Chem. Soc. 2005, 127, 6958-6959. (d) Narasimhaswamy, T.; Somanathan, N.; Lee, D. K.; Ramamoorthy, A. Chem. Mater. 2005, 17, 2013-2018.

(15) (a) Pelzl, G.; Schroder, M. W.; Dunemann, U.; Diele, S.; Weissflog, W.; Jones, C.; Coleman, D.; Clark, N. A.; Stannarius, R.; Li, J. J.; Das, B.; Grande, S. J. Mater. Chem. 2004, 14, 2492-2498. (b) Elmahdy, M. M.; Floudas, G.; Mondeshki, M.; Spiess, H. W.; Dou, X.; Mullen, K. Phys. Rev. Lett. 2008, 100, 107801. (c) Khitrin, A. K.; Ermakov, V. L.; Fung, B. M. J. Chem. Phys. 2002, 117, 6903-6906. (d) Severing, K.; StibalFischer, E.; Hasenhindl, A.; Finkelmann, H.; Saalwachter, K. J. Phys. Chem. 2006, 110, 15680-15688. (e) Xu, J.; Dong, R. Y.; Domenici, V.; FodorCsorba, K.; Veracini, C. A. J. Phys. Chem. B 2006, 110, 9434-9441. (f) Xu, J.; Fodor-Csorba, K.; Dong, R. Y. J. Phys. Chem. A 2005, 109, 19982005 
(16) Hartmann, S. R.; Hahn, E. L. Phys. Rev. 1962, 128, 2042-2053. (17) Pines, A.; Gibby, M. G.; Waugh, J. S. J. Chem. Phys. 1973, 59, 569-590.

(18) Schaefer, J.; Stejskal, E. O. J. Am. Chem. Soc. 1976, 98, 10311032.

(19) Bennett, A. E.; Rienstra, C. M.; Auger, M.; Lakshmi, K. V.; Griffin, R. G. J. Chem. Phys. 1995, 103, 6951-6958. 486.

(20) Morcombe, C. R.; Zilm, K. W. J. Magn. Reson. 2003, 162, 479-

(21) Bak, M.; Rasmussen, J. T.; Nielsen, N. C. J. Magn. Reson. 2000, 147, 296.

(22) Veeman, W. S. Prog. Nucl. Magn. Reson. Spectrosc. 1984, 16, $193-$ 235.

(23) Wang, S.; Gu, M.; Yu, Y.; Xu, X.; Wu, J.; Yuan, G.; Feng, D. Int. J. Mod. Phys. B 2005, 19, 2398-2403.

(24) Courtieu, J.; Bayle, J. P.; Fung, B. M. Progr. NMR Spectrosc. 1994, $26,141-169$

(25) Duncan, T. M. J. Phys. Chem. Ref. Data 1987, 16, 125.

(26) (a) Ramamoorthy, A.; Wei, Y.; Lee, D. K. Annu. Rep. NMR Spectrosc. 2004, 52, 1-52. (b) Ramamoorthy, A.; Yamamoto, K. Modern Magnetic Resonance; Webb, G. A., Ed.; Springer: Dordrecht, 2006; pp 699705 .

(27) Schmidt-Rohr, K.; Nanz, D.; Emsley, L.; Pines, A. J. Phys. Chem. 1994, 98, 6668-6670.

(28) (a) Hester, R. K.; Ackerman, J. L.; Neff, B. L.; Waugh, J. S. Phys. Rev. Lett. 1976, 36, 1081-1083. (b) Wu, C. H.; Ramamoorthy, A.; Opella, S. J. J. Magn. Reson. Ser. A 1994, 109, 270-272. (c) Fu, R. Q.; Tian, C. L.;
Kim, H.; Smith, S. A.; Cross, T. A. J. Magn. Reson. 2002, 159, 167-174. (d) Nevzorov, A. A.; Opella, S. J. J. Magn. Reson. 2003, 164, 182-186. (e) Fu, R. Q.; Hu, J.; Cross, T. A. J. Magn. Reson. 2004, 168, 8-17. (f) Yamamoto, K.; Lee, D. K.; Ramamoorthy, A. Chem. Phys. Lett. 2005, 407, 289-293. (g) Dvinskikh, S. V.; Yamamoto, K.; Ramamoorthy, A. Chem. Phys. Lett. 2006, 419, 168-173. (h) Yamamoto, K.; Dvinskikh, S. V.; Ramamoorthy, A. Chem. Phys. Lett. 2006, 419, 533-536. (i) Dvinskikh, S. V.; Yamamoto, K.; Ramamoorthy, A. J. Chem. Phys. 2006, 125, 034507.

(29) (a) Lu, J. X.; Damodaran, K.; Lorigan, G. A. J. Magn. Reson. 2006, 178, 283-287. (b) Dvinskikh, S. V.; Dürr, U. H. N.; Yamamoto, K.; Ramamoorthy, A. J. Am. Chem. Soc. 2006, 128, 6326-6327.

(30) Ramamoorthy, A.; Opella, S. J. Solid State NMR Spectrosc. 1995, 4, 387-392.

(31) (a) Caravatti, P.; Bodenhausen, G.; Ernst, R. R. Chem. Phys. Lett. 1982, 89, 363-367. (b) Dvinskikh, S. V.; Zimmermann, H.; Maliniak, A.; Sandström, D. J. Magn. Reson. 2003, 163, 46-55.

(32) Dvinskikh, S. V.; Dürr, U. H. N.; Yamamoto, K.; Ramamoorthy, A. J. Am. Chem. Soc. 2007, 129, 794-802.

(33) Burum, D. P.; Linder, M.; Ernst, R. R. J. Magn. Reson. 1981, 44, 173-188.

(34) Dvinskikh, S. V.; Zimmermann, H.; Maliniak, A.; Sandström, D. J. Magn. Reson. 2004, 168, 194-201.

(35) Zhao, X.; Eden, M.; Levitt, M. H. Chem. Phys. Lett. 2001, 342, $353-361$.

JP803265Z 